\title{
Hereditary hemorrhagic telangiectasia
}

\section{Anne Grand'Maison MD}

$\infty$ See related case by Manawadu and colleagues, page 836, and clinical images by Nanda and Bhatt, page 838, and by Irani and Kasmani, page 839

$\mathrm{H}$ ereditary hemorrhagic telangiectasia, or OslerWeber-Rendu syndrome, is an autosomal dominant vascular disorder that affects multiple systems. It is characterized by skin and mucosal telangiectasias and arteriovenous malformations. In 1876, Sir John Legg described a case of "hemophilia" with recurrent epistaxis and multiple naevi. Rendu, Osler and Weber later contributed to a more comprehensive description of the disease when they reported familial cases of multiple cutaneous telangiectasias and spontaneous epistaxis that were distinct from hemophilia.

Hereditary hemorrhagic telangiectasia occurs worldwide. Its frequency varies between countries, but this is thought to be largely because of underreporting. The estimated prevalence ranges from 1 in 1331 Afro-Caribbean people in the Netherlands Antilles ${ }^{1}$ to 1 in 39216 people in northern England. ${ }^{2}$ The frequency is similar among men and women.

Several mutations in 2 genes, endoglin on chromosome 9 (HHT type 1) and activin receptor-like kinase-1 on chromosome 12 (HHT type 2), are associated with hereditary hemorrhagic telangiectasia. These genes are implicated in vascular development and repair. The gene MADH4 has been linked to hereditary hemorrhagic telangiectasia in association with juvenile polyposis and a new locus on chromosome 5 has recently been identified. ${ }^{3}$ Clinical symptoms vary according to genotype. ${ }^{4}$ Mutations in HHT subtype 1 are associated with a higher frequency of pulmonary arteriovenous malformations. Mutations in HHT subtype 2 are less commonly associated with cerebral and pulmonary arteriovenous malformations than mutations in HHT subtype 1, but there may be a higher frequency of hepatic lesions.

\section{Characteristics and treatment}

Patients can present with a wide spectrum of clinical problems, such as telangiectasias and arteriovenous malformations, caused by abnormal vascular structures. They may also have a family history of hereditary hemorrhagic telangiectasia. ${ }^{5-7}$ HHT Foundation International has developed the Curaçao diagnostic criteria (Box 1), ${ }^{8}$ which can help clinicians determine the probability of hereditary hemorrhagic telangiectasia. Once hereditary hemorrhagic telangiectasia is suspected, appropriate investigations and referrals can be made. ${ }^{7,8}$

\section{Key points}

- Hereditary hemorrhagic telangiectasia is underrecognized.

- Patients with hereditary hemorrhagic telangiectasia require annual monitoring for new telangiectasias and arteriovenous malformations and for complications related to existing lesions.

- Treatments such as embolization, laser therapy or surgery may be required for telangiectasias or visceral lesions.

- Many patients will require regular replenishment of iron stores.

- Relatives of patients with hereditary hemorrhagic telangiectasia should be screened for the condition.

\section{Mucocutaneous telangiectasias \\ Skin and mucosa}

Telangiectasias of the skin and mucosa are common among patients with hereditary hemorrhagic telangiectasia and usually affect the face, lips, tongue, oral mucosa, gums, conjunctiva, trunk, arms and fingers. ${ }^{5-7}$ Lesions tend to occur at a young age and progress as the patient ages. Bleeding from these sites is usually mild and easily contained. Laser ablation can be used as needed.

\section{Nose}

Spontaneous and recurrent epistaxis secondary to nasal telangiectasias ${ }^{5}$ occurs in more than $90 \%$ of patients with hereditary hemorrhagic telangiectasia. This is the most common manifestation. Nasal bleeding, which usually appears early in childhood, ${ }^{7}$ can be mild with no treatment required or severe with transfusions required. ${ }^{6}$ Uncontrolled recurrent epistaxis may cause anemia. ${ }^{5,6}$

Epistaxis can be managed by use of standard treatments (e.g., packing, antifibrinolytics, estrogen or progesterone therapy), laser therapy, surgery or embolization. ${ }^{5}$ No one treatment has been shown to be superior to another. Choices of treatment depend on physicians' preferences and the availability of resources.

\section{Gastrointestinal tract}

Gastrointestinal telangiectasias are found in the large and small intestine in $15 \%-30 \%$ of patients with hereditary hem-

Anne Grand'Maison is a hematologist with the Department of Health Policy Management and Evaluation, University of Toronto, Toronto, Ont. 


\section{Box 1: The Curaçao diagnostic criteria ${ }^{8}$}

The diagnosis of hereditary hemorrhagic telangiectasia is definite if 3 of the following criteria are present, possible or suspected if 2 are present, and unlikely if fewer than 2 are present:

- Epistaxis: spontaneous, recurrent nose bleeds

- Telangiectasias: multiple, at characteristic sites (lips, oral cavity, fingers and nose)

- Visceral lesions: gastrointestinal telangiectasias (with or without bleeding) and arteriovenous malformations (pulmonary, hepatic, cerebral and spinal)

- Family history: a first-degree relative with hereditary hemorrhagic telangiectasia

orrhagic telangiectasia. These are frequent causes of acute or chronic gastrointestinal bleeding and anemia. Bleeding rarely occurs before the patient reaches 30 years of age. Telangiectasias can be seen by endoscopy. ${ }^{6}$

Treatment with estrogen-progesterone (daily oral therapy, $0.05 \mathrm{mg}$ ethinylestradiol and $1 \mathrm{mg}$ norethisterone), ${ }^{9}$ antifibrinolytics and laser therapy may be effective in reducing the severity of bleeding and the necessity for blood transfusions.

Iron-deficiency anemia is a common consequence of uncontrolled bleeding ${ }^{6}$ from the gastrointestinal tract or epistaxis. Infusions of iron must be received on a regular basis to reduce the need for transfusion. For patients intolerant to iron infusion, oral supplements can be taken. Blood transfusions are given when necessary. ${ }^{6}$

\section{Arteriovenous malformations}

These malformations develop most commonly in the lungs, liver and central nervous system.

\section{Pulmonary}

Pulmonary arteriovenous malformations occur in 5\%-30\% of patients aged 30 or more years with hereditary hemorrhagic telangiectasia. Patients may have no symptoms, or they may present with bleeding, such as massive thoracic hemorrhage or hemoptysis. ${ }^{510}$ Patients may also show signs of hypoxemia or orthodeoxia with dyspnea, cyanosis, clubbing and polycythemia-related to a right-to-left shunt. ${ }^{7}$ Stroke and brain abscess secondary to thrombi that originate in the lungs (known as paradoxical emboli) are the most serious consequences of pulmonary arteriovenous malformations. These complications may be the first manifestation of hereditary hemorrhagic telangiectasia.?

Appropriate and timely investigation and treatment are crucial. The investigations for patients with suspected pulmonary malformations include chest radiographs, measurement of the shunt fraction by the $100 \%$ breathing method (calculation of the arterial oxygen tension and of the oxygen saturation after breathing $100 \%$ oxygen for 20 minutes, with a shunt fraction $>5 \%$ defined as abnormal) ${ }^{10}$ and echocardiography to identify the presence of a right-to-left shunt. ${ }^{7}$ A difference in the arterial oxygen tension and in the oxygen saturation in the erect and supine positions (orthodeoxia) could indicate a shunt, but this is a less sensitive screening method. ${ }^{10}$ A chest computed tomography (CT) $s c a n^{6,7}$ or pulmonary angiography ${ }^{5}$ must be performed to confirm and characterize the malformations to determine the appropriate interventions.

Embolization is the treatment of choice and is indicated when the diameter of the feeding artery is at least $3 \mathrm{~mm} .^{5.7,10}$ Surgery is an option for patients who are not candidates for embolization (e.g., untreatable contrast allergy, contraindication to contrast material or if the pulmonary malformations are not amenable to embolization). ${ }^{11}$

Patients with pulmonary arteriovenous malformations (either treated or not) must have regular chest CT scans to detect growth or recanalization of known malformations and to detect new lesions. Screening for pulmonary arteriovenous malformations is also recommended before pregnancy because these malformations tend to enlarge during gestation, which may increase the risk of severe hemorrhage. ${ }^{12}$

\section{Hepatic}

Up to $70 \%$ of patients with hereditary hemorrhagic telangiectasia will develop hepatic arteriovenous malformations, which are often silent. ${ }^{5,13}$ These may lead to high output congestive heart failure, portal hypertension and, rarely, liver failure with or without encephalopathy. Vascular lesions are found by angiography, CT, ${ }^{6}$ magnetic resonance imaging (MRI) or Doppler sonography.

Embolization of hepatic malformations carries a high risk of complications and is not generally recommended. Liver transplantation may be necessary for patients with acute hepatic failure.

\section{Cerebral and spinal}

Neurologic problems occur in $10 \%-15 \%$ of patients with hereditary hemorrhagic telangiectasia. These complications tend to occur later in life. They include transient ischemic attack, ischemic stroke and brain abscess related to pulmonary arteriovenous malformations with right-to-left shunting.? Cerebral and spinal malformations can cause devastating hemorrhage and are best detected by MRI.

Treatment includes embolization of pulmonary lesions and microsurgery, radiation therapy or embolization of cerebral malformations. Screening for neurologic lesions in asymptomatic patient with hereditary hemorrhagic telangiectasia is often recommended ${ }^{7}$ but is controversial. ${ }^{14}$

\section{Surveillance}

Monitoring is recommended for patients with a definite diagnosis of hereditary hemorrhagic telangiectasia. ${ }^{15}$ It should include the following:

- Annual visit with a physician familiar with hereditary hemorrhagic telangiectasia. This visit should include a thorough history and physical examination for new mucocutaneous telangiectasias, epistaxis, gastrointestinal bleeding, chest symptoms (e.g., shortness of breath, reduction in exercise tolerance, hemoptysis) and neurologic symptoms.

- Annual testing for occult blood in stool.

- Annual complete blood count to detect new-onset anemia, 
and more frequent testing based on the patient's bleeding history.

- Pulse oximetry in the supine and sitting positions every 1-2 years during childhood (to screen for pulmonary arteriovenous malformations); a contrast echocardiogram should be obtained if the oxygen saturation is below $97 \%$, followed by a chest CT scan if the result of the echocardiogram is positive.

- Contrast echocardiogram to screen for pulmonary shuntting for patients aged 10 years and older, followed by a chest $\mathrm{CT}$ if a shunt is detected.

- Arterial blood gases or a chest CT, or both, about 1 year after embolization to exclude reperfusion of previously treated pulmonary arteriovenous malformations. ${ }^{16}$

- Chest CT scan every 3-5 years for patients previously found to have pulmonary arteriovenous malformation, to reassess the growth of vascular lesions and to exclude the presence of new pulmonary arteriovenous malformations.

- Liver screening for arteriovenous malformation does not need to be routinely performed.

- Cerebral MRI to exclude cerebral arteriovenous malformation is recommended on 1 occasion after the diagnosis of hereditary hemorrhagic telangiectasia has been made.

\section{Family screening}

For many patients with hereditary hemorrhagic telangiectasia, the clinical symptoms appear later in life. Therefore, screening family members for signs of hereditary hemorrhagic telangiectasia is reasonable and should include a complete history, physical examination (including a referral to otolaryngology if possible), chest radiography and arterial blood gas testing (with measurement of the shunt fraction). ${ }^{17}$

Genetic testing for family members of patients with hereditary hemorrhagic telangiectasia can be performed, particularly if a specific gene mutation has been identified for the affected patient or if the family member is at high risk for severe complications. However, a negative result does not exclude the disease. ${ }^{18}$

\section{Conclusion}

Hereditary hemorrhagic telangiectasia is a vascular disorder with myriad manifestations. Despite a better understanding of the disease, hereditary hemorrhagic telangiectasia is still not fully appreciated by clinicians, who often do not recognize the disorder until severe or even life-threatening manifestations occur. ${ }^{7} \mathrm{~A}$ higher level of awareness is necessary to diagnose this disease early and improve outcomes. A referral to a treatment centre for hereditary hemorrhagic telangiectasia is advisable for complete individual and family evaluation, treatment and education.
Competing interests: None declared.

\section{REFERENCES}

1. Westermann CJ, Rosina AF, De Vries, V, et al. The prevalence and manifestations of hereditary hemorrhagic telangiectasia in the Afro-Caribbean population of the Netherlands Antilles: a family screening. Am J Med Genet A 2003;116A:324-8.

2. Porteous ME, Burn J, Proctor SJ. Hereditary haemorrhagic telangiectasia: a clinical analysis. J Med Genet 1992;29:527-30.

3. Prigoda NL, Savas S, Abdalla SA, et al. Hereditary haemorrhagic telangiectasia: mutation detection, test sensitivity and novel mutations. J Med Genet 2006; 43:722-8.

4. Letteboer TG, Mager HJ, Snijder RJ, et al. Genotype-phenotype relationship for localization and age distribution of telangiectases in hereditary hemorrhagic telangiectasia. Am J Med Genet A 2008;146A:2733-9.

5. Nada S, Bhatt SP. Hereditary hemorrhagic telangiectasia: epistaxis and hemoptysis. CMAJ 2009; 180:838.

6. Irani F, Kasmani R. Hereditary hemorrhagic telangiectasia: fatigue and dyspnea. CMAJ 2009; 180:839.

7. Manawadu D, Vethanayagam D, Ahmed SN. Hereditary hemorrhagic telangiectasia: transient ischemic attacks. CMAJ 2009;180:836-7.

8. Shovlin CL, Guttmacher AE, Buscarini E, et al. Diagnostic criteria for hereditary hemorrhagic telangiectasia (Rendu-Osler-Weber syndrome). Am J Med Genet 2000;91:66-7.

9. van Cutsem E, Rutgeerts P, Vantrappen G. Treatment of bleeding gastrointestinal vascular malformations with oestrogen-progesterone. Lancet 1990;335:953-5.

10. Shovlin CL, Letarte M. Hereditary haemorrhagic telangiectasia and pulmonary arteriovenous malformations: issues in clinical management and review of pathogenic mechanisms. Thorax 1999;54:714-29.

11. Gossage JR, Kanj G. Pulmonary arteriovenous malformations. A state of the art review. Am J Respir Crit Care Med 1998;158:643-61.

12. Shovlin CL, Sodhi V, McCarthy A, et al. Estimates of maternal risks of pregnancy for women with hereditary haemorrhagic telangiectasia (OslerWeber-Rendu syndrome): suggested approach for obstetric services. BJOG 2008; $115: 1108-15$

13. Memeo M, Stabile Ianora AA, Scardapane A, et al. Hepatic involvement in hereditary hemorrhagic telangiectasia: CT findings. Abdom Imaging 2004;29:211-20.

14. Easey AJ, Wallace GM, Hughes JM, et al. Should asymptomatic patients with hereditary haemorrhagic telangiectasia (HHT) be screened for cerebral vascular malformations? Data from 22,061 years of HHT patient life. J Neurol Neurosurg Psychiatry 2003;74:743-8.

15. Bayrak-Toydemir P, Mao R, Lewin S, et al. Hereditary hemorrhagic telangiectasia: an overview of diagnosis and management in the molecular era for clinicians. Genet Med 2004;6:175-91.

16. Lee DW, White RI Jr, Egglin TK, et al. Embolotherapy of large pulmonary arteriovenous malformations: long-term results. Ann Thorac Surg 1997;64:930-9.

17. Cottin V, Plauchu H, Bayle JY, et al. Pulmonary arteriovenous malformations in patients with hereditary hemorrhagic telangiectasia. Am J Respir Crit Care Med 2004;169:994-1000.

18. Haitjema T, Disch F, Overtoom TT, et al. Screening family members of patients with hereditary hemorrhagic telangiectasia. Am J Med 1995;99:519-24.

\author{
Resources \\ HHT Foundation International \\ www.hht.org \\ Tel 800 448-6389, 410 357-9932 \\ Toronto HHT Centres \\ www.hhttoronto.com \\ Tel $416864-6060$ ext. 2407 \\ Edmonton HHT Centre \\ Tel 780 407-1479 \\ Montréal HHT Centre \\ Tel 514 890-8000 ext. 15672
}

